\title{
POSITIVE SOLUTIONS OF ELLIPTIC AND PARABOLIC EQUATIONS WITH CONVEX-CONCAVE NONLINEARITIES
}

\author{
QIUYi DAI AND YongGeng GU
}

(Received October 23, 2003, revised July 21, 2004)

\begin{abstract}
We consider, respectively, the Dirichlet problem and the initial-boundary value problem of elliptic and parabolic equations with two power nonlinearities. We find that these problems are closely related to the so-called quenching problem. We obtain the existence and nonexistence of positive solutions to these problems on bounded and unbounded domains, by using the results of quenching problem and sub-super solution method.
\end{abstract}

1. Introduction. Let $\Omega$ be an arbitrary domain in Euclidean $n$-space $\boldsymbol{R}^{n}$. We consider the Dirichlet problem of the semi-linear elliptic equation

$$
\begin{cases}-\Delta u=u^{p}+u^{q}, & x \in \Omega, \\ u=0, & x \in \partial \Omega,\end{cases}
$$

and the initial-boundary value problem of its parabolic version

$$
\begin{cases}\frac{\partial u}{\partial t}-\Delta u=u^{p}+u^{q}, & (x, t) \in \Omega \times(0, T), \\ u=0, & (x, t) \in \partial \Omega \times(0, T), \\ u(x, 0)=u_{0}(x), & x \in \Omega .\end{cases}
$$

In most cases, we assume that $0 \leq q<1<p$. When $\Omega$ is a bounded domain, problems similar to (1.1) were studied by many authors. If $1 \leq q<p \leq(n+2) /(n-2)$, we refer to [18-20] and the references cited therein. If $0 \leq q<1<p$, we refer to Ambrosetti, Brezis and Cerami [1], Boccard, Escobedo and Peral [2], Jin [3], Bartsch and Willem [4], Cabre and Majer [5] and so on. Problems similar to (1.2) were studied by Cazenave, Dickstein and Escobedo [6]. Compared to these circumstances, there are few results for Problems (1.1) and (1.2) on unbounded domains. This motivates us to study Problems (1.1) and (1.2) on unbounded domains in the present paper. Our main focus is the existence and nonexistence of positive solutions to Problem (1.1), as well as the existence and nonexistence of global solutions to Problem (1.2). However, we find that to accomplish this task, some new results for Problems (1.1) and (1.2) on bounded domains are needed. Accordingly, we have to first revisit Problems (1.1) and (1.2) on bounded domains.

The paper is organized as follows. In Section 2, we will study Problem (1.1) on bounded domains in the case $0 \leq q<1<p$ and show that there exist two positive numbers $a^{*}$ and $a_{*}$ such that Problem (1.1) has at least one positive solution for $\lambda_{1}(\Omega) \geq a^{*}$, whereas has

2000 Mathematics Subject Classification. Primary 35J25; Secondary 35K20.

Key words and phrases. Elliptic equation, parabolic equation, quenching problem, Dirichlet problem, initialboundary value problem, positive solution.

This work is supported by NNSF of China (Grant: No.10171029). 
no positive solution for $\lambda_{1}(\Omega)<a_{*}$. Here, $\lambda_{1}(\Omega)$ is the first eigenvalue of the eigenvalue problem

$$
\begin{cases}-\Delta \varphi=\lambda \varphi, & x \in \Omega, \\ \varphi=0, & x \in \partial \Omega .\end{cases}
$$

We emphasis here that our results differ from these available so far; for details we refer to Conclusion 2.3 and Example 2.4 in Section 2. In Section 3, we will study Problem (1.1) on unbounded domains and prove that if the in-radius of the domain $\Omega$ is infinite, then Problem (1.1) has no positive solution; while, if the in-radius of the domain $\Omega$ is finite, then Problem (1.1) has at least one positive solution under some suitable conditions. It is worth pointing out that these conditions may be satisfied by some Esteban-Lions domains. Hence, the presence of the sub-linear term $u^{q}$ tremendously changes the structure of the solution set of Problem (1.1) (see Example 3.8). In Section 4, we will study Problem (1.2) on bounded domains and prove that it has a global solution for some suitable initial value $u_{0}(x)$ provided that $\lambda_{1}(\Omega)$ is large enough, while it has no global solution for any nonnegative initial value $u_{0}(x)$ if $\lambda_{1}(\Omega)$ is small enough. This result is different to some extent from the result given in [6]. In Section 5, we will study Problem (1.2) with $\Omega=\boldsymbol{R}^{n}$, i.e., the Cauchy problem. The main contribution of this section is a computation of the critical exponent of Fujita's type. In Section 6, we state a result for Problem (1.2) on general unbounded domains.

2. Elliptic Equation on Bounded Domains. Let $\Omega \subseteq \boldsymbol{R}^{n}$ be a bounded domain. In this section, we investigate the following Dirichlet problem

$$
\begin{cases}-\Delta u=u^{p}+u^{q}, & x \in \Omega, \\ u>0, & x \in \Omega, \\ u=0, & x \in \partial \Omega .\end{cases}
$$

Since the case of $1 \leq p, q \leq(n+2) /(n-2)$ is well studied in recent years, we are mainly interested in the case $0 \leq q<1<p$. Let $\lambda_{1}(\Omega)$ denote the first eigenvalue of the eigenvalue Problem (1.3). The main result of this section can be stated as

THEOREM 2.1. (i) If $\lambda_{1}(\Omega)<[(1-q) /(p-1)]^{(p-1) /(p-q)}+[(1-q) /(p-$ $1)]^{(q-1) /(p-q)}$, then Problem (2.1) has no solution.

(ii) If $\lambda_{1}(\Omega) \geq C_{0}(n)$, then Problem (2.1) has at least one solution, where $C_{0}(n)=$ $16(n / \pi e)^{n / 4}\left(\omega_{n-1} / n\right)^{1 / 2}+8(2 / \pi)^{n / 2} \omega_{n-1} \Gamma(n / 2), \omega_{n-1}$ is the area of unit sphere in $\boldsymbol{R}^{n}$ and $\Gamma(x)$ is the Gamma function.

Before proving Theorem 2.1, some remarks are in order.

REMARK 2.2. The nonlinear term $g(u)=u^{p}+u^{q}$ in the differential equation of Problem (2.1) satisfies the conditions imposed in [3]. Hence, a typical result obtained in [3] in the present context is

CONCLUSION 2.3 ([3]). There exists a positive number $\Lambda(n, p, q)$ depending only on $n, p$ and $q$ such that Problem (2.1) has at least one solution, provided that $|\Omega|<\Lambda(n, p, q)$. Here $|\Omega|$ denotes Lebesgue's measure of $\Omega$. 
It is worth pointing out that there is a significant difference between the Conclusion 2.3 and our result Theorem 2.1(ii). This may be illustrated by the following example.

EXAMPLE 2.4. Let $B_{a}^{n-1}(0)$ denote the ball in $\boldsymbol{R}^{n-1}$ with radius $a$ and center $o$. Let $\mu_{1}(a)$ be the first eigenvalue of the eigenvalue problem

$$
\begin{cases}\Delta \varphi+\mu \varphi=0, & x \in B_{a}^{n-1}(0), \\ \varphi=0, & x \in \partial B_{a}^{n-1}(0) .\end{cases}
$$

We choose $a_{0}$ so small that $\mu_{1}\left(a_{0}\right) \geq C_{0}(n)$ and set $\Omega=B_{a_{0}}^{n-1} \times(0, b)$ in Problem (2.1). It is obvious that $|\Omega|>\Lambda(n, p, q)$ for $b$ large enough, since $|\Omega|=\left|B_{a_{0}}\right| \times b \rightarrow+\infty$ as $b \rightarrow+\infty$. This implies that Conclusion 2.3 cannot apply to verify this example for large $b$. However, by a separation variable method, we can easily see that

$$
\lambda_{1}(\Omega)=\mu\left(a_{0}\right)+\frac{\pi^{2}}{b^{2}}>C_{0}(n) \text { for all } b>0 .
$$

Hence, we may apply Theorem 2.1(ii) to obtain one solution of Problem (2.1) for any $b>0$.

REMARK 2.5. In the case of $\lambda_{1}(\Omega) \geq C_{0}(n)$ and $1<p \leq(n+2) /(n-2)$, one can prove that Problem (2.1) has at least two solutions by an argument similar to that used in Section 4 of [1]. The sub-linear term $u^{q}$ plays an important role in finding the second solution of Problem (2.1).

To prove Theorem 2.1, some lemmas are needed. First, we prove the following

LEMMA 2.6. Let $\lambda$ be a positive number, and $0 \leq q<1<p$. Then we have

$$
\lambda z \leq z^{q}+\left(\frac{\lambda c}{c^{p}+c^{q}}\right)^{(p-q) /(1-q)} z^{p} \text { for all } z \geq 0,
$$

where $c=[(1-q) /(p-1)]^{1 /(p-q)}$.

PROOF. Since the conclusion of Lemma 2.6 is obviously valid for $z=0$, we have only to prove Lemma 2.6 for $z>0$. From now on, let us assume that $z>0$ and set

$$
F(z)=\left(z^{p}+\eta z^{q}\right) /(\lambda z),
$$

where $\eta$ is a positive real number to be determined later.

Since

$$
F^{\prime}(z)=\frac{(p-1) z^{p-q}-\eta(1-q) z^{q}}{\lambda z^{2}},
$$

we can easily find that

$$
\min _{z>0} F(z)=\frac{c^{p}+c^{q}}{\lambda c}, c=\left(\frac{1-q}{p-1}\right)^{1 /(p-q)} .
$$

Moreover, this minimum is attained at $z=c \eta^{1 /(p-q)}$.

Consequently,

$$
\frac{z^{p}+\eta z^{q}}{\lambda z} \geq \frac{c^{p}+c^{q}}{\lambda c} \eta^{(p-1)(p-q)} \text { for all } \quad z>0,
$$


that is,

$$
\lambda z \leq \frac{\lambda c \eta^{(1-q) /(p-q)}}{c^{p}+c^{q}} z^{q}+\frac{\lambda c}{c^{p}+c^{q}}\left(\frac{1}{\eta}\right)^{(p-1) /(p-q)} z^{p} .
$$

Choosing $\eta$ suitably so that

$$
\frac{\lambda c \eta^{(1-q) /(p-q)}}{c^{p}+c^{q}}=1
$$

we get

$$
\lambda z \leq z^{q}+\left[\frac{\lambda c}{c^{p}+c^{q}}\right]^{(p-q) /(1-q)} z^{p} \text { for all } z>0 .
$$

This completes the proof of Lemma 2.6.

Next, we consider the initial-boundary value problem

$$
\begin{cases}\frac{\partial v}{\partial t}-\Delta v=\frac{1}{1-v}, & (x, t) \in \Omega \times(0, T), \\ v=0, & (x, t) \in \partial \Omega \times(0, T), \\ v(x, 0)=0, & x \in \Omega,\end{cases}
$$

and its elliptic version

$$
\begin{cases}-\Delta w=\frac{1}{1-w}, & x \in \Omega, \\ w=0, & x \in \partial \Omega .\end{cases}
$$

Problem (2.3) has its own interest. It is the so-called quenching problem and has been studied by many authors since 1976 (see [25]). For the most recent progresses, we refer to Souplet [7], Dai and Gu [21], Dai and Zeng [22] and Dai and Gu [23]. Since previous results for Problems (2.3) and (2.4) do not satisfy the present need, we shall give some results of Problems (2.3) and (2.4) here. The crucial result needed in the proof of Theorem 2.1 is

LEMMA 2.7. Let $C_{0}(n)$ be the same constant as that in Theorem 2.1(ii). If $\lambda_{1}(\Omega) \geq$ $C_{0}(n)$, then Problem (2.4) has at least one classical solution $w(x)$. Moreover, $w(x)$ satisfies $0<w(x) \leq 1 / 2$ for all $x \in \Omega$.

To prove Lemma 2.7, we will need the following lemma.

LEMMA 2.8. Let $\left(e^{t \Delta}\right)_{t \geq 0}$ be the heat semigroup on $\Omega$ with the Dirichlet boundary condition, and $\left|e^{t \Delta}\right|_{\infty}=\left\|e^{t \Delta}\right\|_{\mathscr{L}\left(L^{\infty}(\Omega)\right)}$ denote the norm of $e^{t \Delta}$ as an operator from $L^{\infty}(\Omega)$ into itself. Let $\lambda_{1}(\Omega)$ be the first eigenvalue of the eigenvalue Problem (1.3). Then the estimate

$$
\left|e^{t \Delta}\right|_{\infty} \leq \frac{1}{16} C_{0}(n) e^{-\lambda_{1}(\Omega) t / 4}
$$

is valid. Here $C_{0}(n)$ is the same constant as that in Lemma 2.7.

REMARK 2.9. Lemma 2.8 is essentially proved in Souplet [7] except an explicit formula of $C_{0}(n)$. To obtain an explicit expression of the constant $C_{0}(n)$ and for the completeness of this paper, we give a proof of Lemma 2.8 here. 
PROOF OF LEMMA 2.8. Let $I$ be an arbitrary function defined on $\Omega$ whose sup-norm equals 1. For fixed $t>0$ and $x \in \Omega$, let $B(x, \rho)=\left\{y \in \boldsymbol{R}^{n} ;|x-y|<\rho\right\}$ and $\omega=$ $\Omega \cap B(x, \rho)$. If we denote by $I_{\omega}$ and $I_{\Omega \backslash \omega}$ the restrictions of $I$ on $\omega$ and $\Omega \backslash \omega$ respectively, then $e^{t \Delta} I(x)$ can be written as

$$
e^{t \Delta} I(x)=e^{t \Delta} I_{\omega}(x)+e^{t \Delta} I_{\Omega \backslash \omega}(x) .
$$

First, by the semigroup property and the $L^{2}-L^{\infty}$ estimate, we have

$$
\begin{aligned}
\left|e^{t \Delta} I_{\omega}\right|_{\infty} & \leq(2 \pi t)^{-n / 4}\left\|e^{t / 2 \Delta} I_{\omega}\right\|_{L^{2}(\Omega)} \\
& \leq(2 \pi t)^{-n / 4}\left\|I_{\omega}\right\|_{L^{2}(\Omega)} e^{-\lambda_{1}(\Omega) t / 2} \\
& \leq(2 \pi)^{-n / 4}\left(\omega_{n-1} / n\right)^{1 / 2}\left(\rho^{2} / t\right)^{n / 4} e^{-\lambda_{1}(\Omega) t / 2}
\end{aligned}
$$

On the other hand, by comparing with the solution of the heat equation in the whole space, we have

$$
\begin{aligned}
e^{t \Delta} I_{\Omega \backslash \omega}(x) & \leq(4 \pi t)^{-n / 2} \int_{|x-y|>\rho} e^{-|x-y|^{2} /(4 t)} d y \\
& =\pi^{-n / 2} \int_{|z|>\rho / \sqrt{4 t}} e^{-|z|^{2}} d z=\pi^{-n / 2} \omega_{n-1} \int_{\rho / \sqrt{4 t}}^{\infty} r^{n-1} e^{-r^{2}} d r \\
& \leq \frac{1}{2}(2 / \pi)^{n / 2} \omega_{n-1} \Gamma(n / 2) e^{-\rho^{2} /(8 t)} .
\end{aligned}
$$

By choosing $\rho=t \sqrt{2 \lambda_{1}(\Omega)}$, it follows that

$$
e^{t \Delta} I(x) \leq C_{1}(n)\left(\lambda_{1}(\Omega) t\right)^{n / 4} e^{-\lambda_{1}(\Omega) t / 2}+C_{2}(n) e^{-\lambda_{1}(\Omega) t / 4},
$$

where

$$
C_{1}(n)=\pi^{-n / 4}\left(\omega_{n-1} / n\right)^{1 / 2}, \quad C_{2}(n)=\frac{1}{2}(2 / n)^{n / 2} \omega_{n-1} \Gamma(n / 2)
$$

Since

$$
\left(\lambda_{1}(\Omega) t\right)^{n / 4} e^{-\lambda_{1}(\Omega) t / 4} \leq n^{n / 4} e^{-n / 4}
$$

we have

$$
\begin{aligned}
e^{t \Delta} I(x) & \leq\left[C_{1}(n)(n / e)^{n / 4}+C_{2}(n)\right] e^{-\lambda_{1}(\Omega) t / 4} \\
& =\frac{1}{16} C_{0}(n) e^{-\lambda_{1}(\Omega) t / 4} .
\end{aligned}
$$

Since $t$ and $x$ are arbitrary, we finally get

$$
\left|e^{t \Delta} I\right|_{\infty} \leq \frac{1}{16} C_{0}(n) e^{-\lambda_{1}(\Omega) t / 4} .
$$

This completes the proof of Lemma 2.8.

With Lemma 2.8 established, we can prove

LEMmA 2.10. If $\lambda_{1}(\Omega) \geq C_{0}(n)$, then Problem (2.3) has a unique global solution $v(x, t)$ such that $0<v(x, t) \leq 1 / 2$ for $(x, t) \in \Omega \times(0,+\infty)$. 
PROOF. It is easy to see that the mild solution of Problem (2.3) is

$$
v(t)=\int_{0}^{t} e^{(t-s) \Delta} \frac{d s}{1-v(s)} .
$$

To obtain a global solution of Problem (2.3), we consider the following iterative process

$$
\begin{aligned}
v_{0}(t) & =0, \\
v_{k+1}(t) & =\int_{0}^{t} e^{(t-s) \Delta} \frac{d s}{1-v_{k}(s)},
\end{aligned}
$$

and prove that the sequence $\left\{v_{k}\right\}$ converges to a global solution of Problem (2.3) as $k \rightarrow+\infty$, provided that $\lambda_{1}(\Omega) \geq C_{0}(n)$. Obviously, this can be done by proving that

$$
\begin{aligned}
& \text { (i) } v_{k+1} \geq v_{k} \geq 0 \text { for } k \geq 0, \\
& \text { (ii) } 0 \leq v_{k} \leq \frac{1}{2} \quad \text { for } k \geq 0 .
\end{aligned}
$$

It is easy to see that (i) follows immediately from the comparison principle and the induction method. Hence, we have only to prove (ii). Now, we prove (ii) by the induction method. Since $v_{0}(t)=0<1 / 2$, it suffice to prove that if $v_{k}(t) \leq 1 / 2$, then $v_{k+1}(t) \leq 1 / 2$. Noting that

$$
v_{k+1}(t)=\int_{0}^{t} e^{(t-s) \Delta} \frac{d s}{1-v_{k}(s)},
$$

we have

$$
v_{k+1}(t) \leq 2 \int_{0}^{t}\left|e^{(t-s) \Delta}\right|_{\infty} d s .
$$

By Lemma 2.8, we also have

$$
\begin{aligned}
v_{k+1}(t) & \leq \frac{C_{0}(n)}{8} \int_{0}^{t} e^{-\lambda_{1}(\Omega)(t-s) / 4} d s \\
& =\frac{C_{0}(n)}{2 \lambda_{1}(\Omega)}\left(1-e^{-\lambda_{1}(\Omega) t / 4}\right) \leq \frac{C_{0}(n)}{2 \lambda_{1}(\Omega)} .
\end{aligned}
$$

Taking the assumption $\lambda_{1}(\Omega) \geq C_{0}(n)$ into account, we finally obtain

$$
v_{k+1}(t) \leq 1 / 2 \text {. }
$$

This completes the proof of Lemma 2.10.

Proof OF LEMma 2.7. Let $v(x, t)$ be the solution of Problem (2.3) obtained in Lemma 2.10. First, we may conclude that $v(x, t)$ is monotonically increasing with respect to $t$, and that there exists a integrable function $w(x)$ such that $w(x)=\lim _{t \rightarrow+\infty} v(x, t)$ and $w(x)=0$ for $x \in \partial \Omega$.

Indeed, for any $h>0$, the auxiliary function

$$
H(x, t)=v(x, t+h)-v(x, t)
$$


satisfies

$$
\begin{cases}\frac{\partial H}{\partial t}-\Delta H=a(x, t) H, & (x, t) \in \Omega \times(0,+\infty), \\ H(x, t)=0, & (x, t) \in \partial \Omega \times(0,+\infty), \\ H(x, 0) \geq 0, & x \in \Omega .\end{cases}
$$

Hence, the maximum principle of parabolic equations yields that

$$
H(x, t) \geq 0 .
$$

This implies that $v(x, t)$ is monotonically increasing with respect to $t$, since $h$ is arbitrary. Moreover, noting that $0 \leq v(x, t) \leq 1 / 2$, we know that $v(x, t)$ converges point-wisely on $\Omega$ as $t \rightarrow+\infty$. Let $w(x)=\lim _{t \rightarrow+\infty} v(x, t)$. It follows that $w(x)$ is integrable on $\Omega$. Furthermore, we also have $\left.w(x)\right|_{\partial \Omega}=0$, since $v(x, t) \equiv 0$ for any $(x, t) \in \partial \Omega \times(0,+\infty)$.

Next, we conclude that $w(x)$ is a solution of Problem (2.4) and thus complete the proof of Lemma 2.7.

Indeed, from the above discussion, we know that $w(x)$ is integrable and satisfies

$$
0 \leq w(x) \leq 1 / 2,\left.\quad w(x)\right|_{\partial \Omega}=0 .
$$

So, we have to prove that $w(x)$ satisfies

$$
-\Delta w(x)=\frac{1}{1-w(x)}, \quad x \in \Omega .
$$

To this end, let $G=G(x, y)$ be Green's function of the Laplace operator on $\Omega$ with the zero Dirichlet boundary condition. Multiplying the differential equation in Problem (2.3) by $G$ and then integrating on $\Omega$, we obtain

$$
\frac{d}{d t} \int_{\Omega} G v(y, t) d y+v(x, t)=\int_{\Omega} \frac{G}{1-v(y, t)} d y .
$$

Integrating the above equation on $[t, t+1]$ with respect to $t$, we get

$$
\int_{\Omega} G[v(y, t+1)-v(y, t)] d y+\int_{t}^{t+1} v(x, \tau) d \tau=\int_{t}^{t+1} \int_{\Omega} \frac{G}{1-v(y, \tau)} d y d \tau .
$$

By the mean value theorem, we know that there exist $\xi_{1}, \xi_{2} \in[t, t+1]$ such that

$$
\int_{\Omega} G[v(y, t+1)-v(y, t)] d y+v\left(x, \xi_{1}\right)=\int_{\Omega} \frac{G}{1-v\left(y, \xi_{2}\right)} d y .
$$

Letting $t \rightarrow+\infty$ on the both sides of the above equality, we obtain

$$
w(x)=\int_{\Omega} \frac{G}{1-w(y)} d y,
$$

which implies that

$$
-\Delta w(x)=\frac{1}{1-w(x)}, \quad x \in \Omega .
$$

Summarizing the above discussion, we complete the proof of Lemma 2.7. 
Proof of TheOrem 2.1. Let $\lambda_{1}(\Omega)$ be the first eigenvalue of the eigenvalue Problem (1.3), and $\varphi_{1}(x)$ the corresponding eigenfunction which is chosen so that $\varphi_{1}(x)>0$ for $x \in \Omega$.

First, we prove (i) of Theorem 2.1. To this end, multiplying the differential equation in Problem (2.1) by $\varphi_{1}(x)$ and then integrating on $\Omega$, we obtain

$$
\lambda_{1}(\Omega) \int_{\Omega} u \varphi_{1} d x=\int_{\Omega} u^{p} \varphi_{1} d x+\int_{\Omega} u^{q} \varphi_{1} d x .
$$

Employing the conclusion of Lemma 2.6, we deduce that

$$
\left[1-\left(\frac{\lambda_{1}(\Omega) c}{c^{p}+c^{q}}\right)^{(p-q) /(1-q)}\right] \int_{\Omega} u^{p} \varphi_{1} d x \leq 0 \quad \text { and } \quad c=\left(\frac{1-q}{p-1}\right)^{1 /(p-q)} .
$$

Suppose that $u(x)$ be a solution of Problem (2.1). We then have

$$
\int_{\Omega} u^{p} \varphi_{1} d x>0
$$

It follows from (2.5) and (2.6) that

$$
\lambda_{1}(\Omega) \geq c^{p-1}+c^{q-1} .
$$

This implies that Problem (2.1) has no solution for

$$
\lambda_{1}(\Omega)<\left(\frac{1-q}{p-1}\right)^{(p-1) /(p-q)}+\left(\frac{1-q}{p-1}\right)^{(q-1) /(p-q)} .
$$

The proof of Theorem 2.1(i) is completed.

Next, we are going to prove (ii) of Theorem 2.1. Since $0 \leq q<1$, it is easy to see that

$$
-\Delta\left(\varepsilon \varphi_{1}(x)\right)=\varepsilon \lambda_{1}(\Omega) \varphi_{1}(x) \leq \varepsilon^{p} \varphi_{1}^{p}(x)+\varepsilon^{q} \varphi_{1}^{q}(x)
$$

for all $\varepsilon>0$ small enough. Hence, $\varepsilon \varphi_{1}(x)$ is a sub-solution of Problem (2.1), provided that $\varepsilon$ is sufficiently small. On the other hand, by the assumption $\lambda_{1}(\Omega) \geq C_{0}(n)$ and Lemma 2.7, we know that there exists a function $w(x)$ satisfying $0 \leq w(x) \leq 1 / 2,\left.\quad w(x)\right|_{\partial \Omega}=0$ and

$$
-\Delta w(x)=\frac{1}{1-w(x)}=1+w(x)+w^{2}(x)+\cdots \geq w^{p}(x)+w^{q}(x) .
$$

This means that $w(x)$ is a super-solution of Problem (2.1). Moreover, we can choose $\varepsilon>0$ so small that

$$
\varepsilon \varphi_{1}(x) \leq w(x) .
$$

Now, by the sub-super-solution method (see [24]), we know that Problem (2.1) has at least one solution $u(x)$, provided that $\lambda_{1}(\Omega) \geq C_{0}(n)$ and $\varepsilon \varphi_{1}(x) \leq u(x) \leq w(x)$. This completes the proof of Theorem 2.1(ii).

REMARK 2.11. The conclusion (ii) of Theorem 2.1 can be generalized to a little more complicated problem such as

$$
\begin{cases}-\Delta u=f(u), & x \in \Omega, \\ u>0, & x \in \Omega, \\ u=0, & x \in \partial \Omega,\end{cases}
$$


where $f(s)$ is a continuous function defined on $[0,+\infty)$ satisfying

$\left(F_{1}\right) \quad \lim _{s \rightarrow 0^{+}} f(s) / s>\lambda_{1}(\Omega)$, and

$\left(F_{2}\right) \quad$ there exist two positive constants $M_{1}$ and $M_{2}$ such that

$$
f(s) \leq M_{1} s^{p}+M_{2} s^{q} \text { for } s \geq 0, \quad 0 \leq q<1<p .
$$

Indeed, $\left(F_{1}\right)$ implies that $\varepsilon \varphi_{1}(x)$ is a sub-solution of Problem (2.7) for $\varepsilon$ sufficiently small. Hence, to obtain a solution of Problem (2.7), it suffices to find a super-solution $w(x)$ of Problem (2.7) with property $\varepsilon \varphi_{1}(x) \leq w(x)$. To this end, we consider the problem

$$
\begin{cases}-\Delta w=M_{1} w^{p}+M_{2} w^{q}, & x \in \Omega, \\ w>0, & x \in \Omega, \\ w=0, & x \in \partial \Omega .\end{cases}
$$

It follows from $\left(F_{2}\right)$ that any solution of Problem (2.8) is a super-solution of Problem (2.7). To solve Problem (2.8), we transform

$$
w(x)=\left(\frac{M_{2}}{M_{1}}\right)^{1 /(p-q)} h(x), \quad y=M_{2}^{(p-1) /\{2(p-q)\}} M_{1}^{(1-q) /\{2(p-q)\}} x,
$$

and set

$$
\begin{aligned}
v(x) & =h\left(M_{2}^{-(p-1) /\{2(p-q)\}} M_{1}^{-(1-q) /\{2(p-q)\}} y\right)=h(x), \\
\tilde{\Omega} & =\left\{y=M_{2}^{(p-1) /\{2(p-q)\}} M_{1}^{(1-q) /\{2(p-q)\}} x \mid x \in \Omega\right\} .
\end{aligned}
$$

Then it is easy to check that Problem (2.8) is transformed into

$$
\begin{cases}-\Delta v=v^{p}+v^{q}, & y \in \tilde{\Omega}, \\ v(y)>0, & y \in \tilde{\Omega}, \\ v(y)=0, & y \in \partial \tilde{\Omega} .\end{cases}
$$

By Theorem 2.1(ii), we know that if $\lambda_{1}(\tilde{\Omega}) \geq C_{0}(n)$, then Problem (2.9) has at least one solution $v(x)$. Consequently, Problem (2.8) has at least one solution $w(x)$ under the same condition. Since $\lambda_{1}(\tilde{\Omega})=M_{2}^{-(p-1) /(p-q)} M_{1}^{-(1-q) /(p-q)} \lambda_{1}(\Omega)$, we conclude that Problem (2.8) has at least one solution $w(x)$ provided $\lambda_{1}(\Omega) \geq C_{0}(n) M_{2}^{(p-1) /(p-q)} M_{1}^{(1-q) /(p-q)}$. Moreover, by choosing $\varepsilon>0$ possibly smaller, we have $\varepsilon \varphi_{1}(x) \leq w(x)$. Thus, we have proved

THEOREM 2.12. Let $\left(F_{1}\right)$ and $\left(F_{2}\right)$ hold, and $\lambda_{1}(\Omega) \geq C_{0}(n) M_{2}^{(p-1) /(p-q)} \times$ $M_{1}^{(1-q) /(p-q)}$. Then Problem (2.7) has at least one solution.

REMARK 2.13. In the case of $f(s)=s^{p}+\lambda s^{q}$, Problem (2.7) was studied by Ambrosetti,Brezis and Cerami in [1], who showed the following result.

CONCLUSION 2.14. There exists a positive number $\Lambda$ such that Problem (2.7) with $f(s)=s^{p}+\lambda s^{q}$ has at least one solution for $0<\lambda \leq \Lambda$, but has no solution for $\lambda>\Lambda$.

Using Theorem 2.12 and the method employed to prove Theorem 2.1(i) in Section 2, we can estimate $\Lambda$ as follows. 
COROLlaRY 2.15. For the number $\Lambda$ in Conclusion 2.14, the following estimate is valid

$$
\left(\frac{\lambda_{1}(\Omega)}{C_{0}(n)}\right)^{(p-q) /(p-1)} \leq \Lambda \leq\left(\frac{\lambda_{1}(\Omega)}{c^{p-1}+c^{q-1}}\right)^{(p-q) /(p-1)},
$$

where $c=[(1-q) /(p-1)]^{1 /(p-q)}$ and $0 \leq q<1<p$.

3. Elliptic Equation on Unbounded Domains. Let $\Omega \subseteq \boldsymbol{R}^{n}$ be an arbitrary unbounded domain. We now introduce the following

DEFINITION 3.1. $\Omega$ is called smooth if there exists a sequence of bounded domains $\left\{\Omega_{i}\right\}$ with $C^{2}$ boundary such that

(i) $\Omega_{i+1} \supset \Omega_{i}$,

(ii) $\Omega=\bigcup_{i=1}^{\infty} \Omega_{i}$.

Let $B_{a}^{k}(y)=\left\{x \in \boldsymbol{R}^{k}|| x-y \mid \leq a\right\}$ denote the ball in $\boldsymbol{R}^{k}$ with radius $a$ and center $y$. It is not difficult to see that an infinite cylindrical domain $\Pi_{a}=B_{a}^{n-1}(0) \times(-\infty,+\infty)$, a semi-infinite cylindrical domain $\Pi_{a}^{+}=B_{a}^{n}(0) \cup\left(B_{a}^{n-1}(0) \times[0,+\infty)\right)$ and an infinite strip $\boldsymbol{R}^{n-1} \times(a, b)$ are smooth domains.

DEFINITION 3.2. Let $\Omega$ be an unbounded domain. Let $n(x)$ denote the outward unit normal to $\partial \Omega$ at a point $x \in \partial \Omega . \Omega$ is called an Esteban-Lions domain if there exists an unit vector $X \in \boldsymbol{R}^{n}$ such that $n(x) \cdot X \geq 0$ and $n(x) \cdot X \not \equiv 0$ on $\partial \Omega$.

Obviously, the half space $\boldsymbol{R}_{+}^{n}=\left\{x \in \boldsymbol{R}^{n} \mid x=\left(x_{1}, \ldots, x_{n}\right), x_{n} \geq 0\right\}$ and the semiinfinite cylindrical domain $\Pi_{a}^{+}$are Esteban-Lions domains.

Definition 3.3. Let $\Omega$ be an unbounded domain. Then the in-radius $\rho(\Omega)$ of $\Omega$ is defined by

$$
\rho(\Omega)=\sup \left\{a \mid \text { there is a ball } B_{a}(y) \text { such that } B_{a}(y) \subset \Omega\right\} .
$$

REMARK 3.4. Let $\Omega$ be a bounded domain in $\boldsymbol{R}^{n}$, and set $\Omega^{c}=\boldsymbol{R}^{n} \backslash \Omega$. Then it is easy to see that $\rho\left(\Omega^{c}\right)=+\infty$. If $\Omega_{n-1}$ is a bounded domain in $\boldsymbol{R}^{n-1}$ and $\Omega=\Omega_{n-1} \times$ $(a,+\infty)$, then $\rho(\Omega)<+\infty$.

An important result in [9] implies that there is a strong relation between the finiteness of $\rho(\Omega)$ and the validity of the Poincare inequality. More precisely, we have

CONCLUSION 3.5. There exists a constant $C(\Omega)$ such that

$$
\|u\|_{L^{2}(\Omega)} \leq C(\Omega)\|\nabla u\|_{L^{2}(\Omega)} \quad \text { for all } \quad u \in H_{0}^{1}(\Omega),
$$

if and only if $\rho(\Omega)<+\infty$.

Based on Conclusion 3.5, if $\rho(\Omega)<+\infty$, then we can define

$$
\lambda_{1}(\Omega)=\inf _{\substack{u \in H_{0}^{1}(\Omega) \\ u \neq 0}} \frac{\int_{\Omega}|\nabla u|^{2} d x}{\int_{\Omega} u^{2} d x} .
$$


It is well-known that if $\Omega$ is a bounded domain, then $\lambda_{1}(\Omega)$ can always be attained by a positive function in $H_{0}^{1}(\Omega)$ and is just the first eigenvalue of the Laplace operator on $\Omega$ with zero Dirichlet data. However, for Esteban-Lions domains $\lambda_{1}(\Omega)$ cannot be achieved by any function in $H_{0}^{1}(\Omega)$ due to a result of Esteban and Lions [8].

From now on, unless specified, we assume that $\Omega$ is a smooth unbounded domain and consider the problem

$$
\begin{cases}-\Delta u=u^{p}+u^{q}, & x \in \Omega, \\ u>0, & x \in \Omega, \\ u=0, & x \in \partial \Omega,\end{cases}
$$

where $0 \leq q<1<p$.

The main result we prove in this section is

THEOREM 3.6. Let $C_{0}(n)$ be the same constant as that in Theorem 2.1(ii), $c=[(1-$ $q) /(p-1)]^{1 /(p-q)}$. Then the following hold.

(i) Problem (3.1) has at least one solution on domains such that $\rho(\Omega)<+\infty$ and $\lambda_{1}(\Omega) \geq C_{0}(n)$.

(ii) Problem (3.1) has no solution on domains such that $\rho(\Omega)=+\infty$, or $\rho(\Omega)<+\infty$ and $\lambda_{1}(\Omega)<c^{p-1}+c^{q-1}$.

To understand an important role played by the sub-linear term $u^{q}$ in Problem (3.1), we compare Problem (3.1) with the following problem

$$
\begin{cases}-\Delta u=u^{p}, & x \in \Omega, \\ u>0, & x \in \Omega, \\ u=0, & x \in \partial \Omega,\end{cases}
$$

and examine the following examples.

EXAMPLE 3.7. It is well-known that if $\Omega=\boldsymbol{R}^{n}$ and $p>(n+2) /(n-2)$, then Problem (3.2) has infinitely many solutions. However, the presence of the sub-linear term $u^{q}$ yields an nonexistence result for Problem (3.1), since $\rho\left(\boldsymbol{R}^{n}\right)=+\infty$. Hence, the appearance of the sub-linear term $u^{q}$ destroys the structure of the set of solutions for Problem (3.2).

EXAMPLE 3.8. It is known from [8] that if $\Omega$ is an Esteban-Lions domain, then Problem (3.2) has no solution. However, the conditions imposed in Theorem 3.1(i) can be satisfied by the Esteban-Lions domain $\Pi_{a}^{+}$for sufficiently small $a$. So, the presence of the sub-linear term $u^{q}$ yields an existence result for Problem (3.1) on this kind of domains, and also substantially changes the structure of the set of solutions for Problem (3.2).

Now, we are going to state and prove some lemmas needed in the proof of Theorem 3.6.

LEMMA 3.9. Assume that $\Omega$ be a bounded domain and that $\lambda_{1}(\Omega) \geq C_{0}(n)$. Then Problem (3.1) has a minimal solution $u^{m}(x)$ in the sense that any solution $u(x)$ of Problem (3.1) satisfies $u^{m}(x) \leq u(x)$ for all $x \in \Omega$. Moreover, $0 \leq u^{m}(x) \leq 1 / 2$.

The proof of this lemma is similar to that of lemma 3.4 in [1], so we omit it here. 
LEMma 3.10. Suppose that $\Omega_{i}, i=1,2$, be two bounded domains satisfying $\Omega_{1} \subset$ $\Omega_{2}$, and $u_{1}^{m}(x)$ and $u_{2}^{m}(x)$ be the minimal solution of Problem (3.1) with respect to $\Omega_{1}$ and $\Omega_{2}$, respectively. Then $u_{2}^{m}(x)>u_{1}^{m}(x)$ for any $x \in \Omega_{1}$.

ProOF. Evidently, $u_{2}^{m}(x)$ is a super-solution of Problem (3.1) with respect to $\Omega_{1}$. At the same time, $\varepsilon \varphi_{1}(x)$ is a sub-solution of Problem (3.1) with respect to $\Omega_{1}$. Furthermore, by choosing $\varepsilon$ possibly smaller, we see that $\varepsilon \varphi_{1}(x) \leq u_{2}^{m}(x)$ for $x \in \Omega_{1}$. Thus, by the sub-super solution method, we know that Problem (3.1) with respect to $\Omega_{1}$ has a solution $u(x)$ satisfying $\varepsilon \varphi_{1}(x) \leq u(x) \leq u_{2}^{m}(x)$. It follows from Lemma 3.9 and the strong maximum principle that

$$
u_{1}^{m}(x) \leq u(x)<u_{2}^{m}(x) \text { for } x \in \Omega_{1} .
$$

This is just the desired conclusion.

Proof of TheOrem 3.6. (i) Since $\Omega$ is a smooth unbounded domain, we know by the definition 3.1 that there exists a sequence of $C^{2}$ bounded domains $\left\{\Omega_{i}\right\}_{i=1}^{\infty}$ such that $\Omega_{i} \subset \Omega_{i+1}$ for $i=1,2, \ldots$, and $\bigcup_{i=1}^{\infty} \Omega_{i}=\Omega$. Furthermore, by the assumption that $\lambda_{1}(\Omega) \geq C_{0}(n)$ and the monotonicity of $\lambda_{1}\left(\Omega_{i}\right)$ with respect to $\Omega_{i}$, we have $\lambda_{1}\left(\Omega_{i}\right) \geq C_{0}(n)$. It then follows from Lemma 3.9 and Lemma 3.10 that the problem

$$
\begin{cases}-\Delta u=u^{p}+u^{q}, & x \in \Omega_{i}, \\ u>0, & x \in \Omega_{i}, \\ u=0, & x \in \partial \Omega_{i}\end{cases}
$$

has a minimal solution $u_{i}^{m}(x)$ such that

$\left(P_{1}\right) \quad u_{i}^{m}(x) \leq u_{i+1}^{m}(x), \quad$ for any $x \in \Omega_{i}$,

$\left(P_{2}\right) \quad 0<u_{i}^{m}(x) \leq 1 / 2, \quad$ for any $x \in \Omega_{i}$.

By $\left(P_{2}\right)$ and the regularity theory of elliptic equations, we know from the diagonal convergence method that, up to a sub-sequence, $\left\{u_{i}^{m}(x)\right\}$ converges uniformly in any bounded subdomain of $\Omega$ to a function $u(x)$ which satisfies

$$
\begin{cases}-\Delta u=u^{p}+u^{q}, & x \in \Omega, \\ u=0, & x \in \partial \Omega .\end{cases}
$$

Moreover, it follows from $\left(P_{1}\right)$ that $u(x)>0$ for $x \in \Omega$. Hence, $u(x)$ is a solution of Problem (3.1). The proof of Theorem 3.6(i) is completed.

(ii) By the assumption that $\rho(\Omega)=+\infty$, or $\rho(\Omega)<+\infty$ and $\lambda_{1}(\Omega)<c^{p-1}+c^{q-1}$, we know that there exists a bounded domain $\Omega_{0} \subset \Omega$ such that

$$
\lambda_{1}(\Omega) \leq \lambda_{1}\left(\Omega_{0}\right)<c^{p-1}+c^{q-1} .
$$

We now consider the Dirichlet problem

$$
\begin{cases}-\Delta v=v^{p}+v^{q}, & x \in \Omega_{0}, \\ v>0, & x \in \Omega_{0}, \\ v=0, & x \in \partial \Omega_{0} .\end{cases}
$$

Then it follows from Theorem 2.1(i) that Problem (3.3) has no solution. On the other hand, if Problem (3.1) has at least one solution $u(x)$, then we can conclude from the sub-super solution method that Problem (3.3) has at least one solution. Indeed, for $\varepsilon>0$ small enough, $\varepsilon \varphi_{1}(x)$ 
and $u(x)$ are sub- and super-solution of Problem (3.3) satisfying $\varepsilon \varphi_{1}(x) \leq u(x)$. This is a contradiction, and hence Theorem 3.6(ii) is proved.

4. Parabolic Equation on Bounded Domains. Let $\Omega \subset \boldsymbol{R}^{n}$ be a bounded domain. We consider the following initial-boundary value problem of the semi-linear parabolic equation

$$
\begin{cases}\frac{\partial u}{\partial t}-\Delta u=u^{p}+u^{q}, & (x, t) \in \Omega \times(0, T), \\ u(x, t)=0, & (x, t) \in \partial \Omega \times(0, T), \\ u(x, 0)=u_{0}(x), & x \in \Omega,\end{cases}
$$

where $0<q<1<p$ and $u_{0}(x) \geq 0$.

Problems similar to (4.1) was studied by Cazenave, Diekstein and Escobedo in [6]. Among other things, the following results were shown in their paper.

THEOREM 4.1 ([6]). For any nonnegative function $u_{0}(x) \in L^{\infty}(\Omega)$, Problem (4.1) has a unique positive solution $u(x, t)$ defined on a maximal time interval $\left[0, T_{m}\right]$. Moreover, the following properties hold:

(i) $u(x, t) \in L^{\infty}(\Omega \times(0, T))$ for all $T<T_{m}$.

(ii) $T_{m} \geq T_{0}$, where $T_{0}=1 /\left(M^{p}+M^{q}\right)$, and $M=\left\|u_{0}(x)\right\|_{L^{\infty}}+1$.

(iii) $|u(x, t)| \leq M$ for all $(x, t) \in \Omega \times\left[0, T_{0}\right]$.

(iv) Either $T_{m}=+\infty$, or else $T_{m}<+\infty$, and $\|u(t)\|_{L^{\infty}(\Omega)} \rightarrow+\infty$ as $t \rightarrow T_{m}$.

THEOREM 4.2 ([6]). Let $u_{0}(x) \leq v_{0}(x)$. Suppose that $u(x, t)$ be a sub-solution and that $v(x, t)$ be a positive super-solution of Problem (4.1) on some interval $(0, T)$ satisfying $u(x, 0)=u_{0}(x)$ and $v(x, 0)=v_{0}(x)$. If $u(x, t)$ and $v(x, t)$ are sufficiently regular (i.e., $u(x, t), v(x, t) \in L^{\infty}(\Omega \times(0, T)) \cap C\left([0, T] ; L^{2}(\Omega)\right) \cap L_{l o c}\left((0, T) ; H^{1}(\Omega)\right) \cap$ $\left.W^{1,2}\left((0, T) ; H^{-1}(\Omega)\right)\right)$, and $u(x, t) \leq v(x, t)$ for all $(x, t) \in \partial \Omega \times(0, T)$, then $u(x, t) \leq$ $v(x, t)$ for all $(x, t) \in \Omega \times[0, T)$.

With the help of Theorem 4.1, Theorem 4.2 and results proved in Section 2, we now prove

THEOREM 4.3. Let $\lambda_{1}(\Omega)$ be the first eigenvalue of the eigenvalue Problem (1.3), $C_{0}(n)$ be the same constant as that in Theorem 2.1(ii) and $c=((1-q) /(p-1))^{1 /(p-q)}$. Then the following hold:

(i) If $\lambda_{1}(\Omega)<c^{p-1}+c^{q-1}$, then Problem (4.1) has no positive global solution for any nonnegative initial data $u_{0}(x)$.

(ii) If $\lambda_{1}(\Omega) \geq C_{0}(n)$, then Problem (4.1) has a positive global solution for $u_{0}(x)$ small enough, while has no positive global solution for $u_{0}(x)$ large enough.

Proof. (i) Multiplying the differential equation in Problem (4.1) by $\varphi_{1}(x)$ and then integrating on $\Omega$ with respect to $x$, we obtain

$$
\frac{d}{d t} \int_{\Omega} u \varphi_{1} d x+\lambda_{1}(\Omega) \int_{\Omega} u \varphi_{1} d x=\int_{\Omega} u^{p} d x+\int_{\Omega} u^{q} \varphi_{1} d x .
$$


Using Lemma 2.6, we then have

$$
\lambda_{1}(\Omega) \int_{\Omega} u \varphi_{1} d x \leq \int_{\Omega} u^{q} \varphi_{1} d x+\left(\frac{\lambda_{1}(\Omega)}{c^{p-1}+c^{q-1}}\right)^{(p-q) /(1-q)} \int_{\Omega} u^{p} \varphi_{1} d x .
$$

Employing (4.2), (4.3) and Jensen's inequality, we get

$$
\frac{d}{d t} \int_{\Omega} u \varphi_{1} d x \geq\left[1-\frac{\lambda_{1}(\Omega)}{c^{p-1}+c^{q-1}}\right]\left(\int_{\Omega} u \varphi_{1} d x\right)^{p}
$$

By the assumption that $\lambda_{1}(\Omega)<c^{p-1}+c^{q-1}$, we see that

$$
M=1-\left(\frac{\lambda_{1}(\Omega)}{c^{p-1}+c^{q-1}}\right)^{(p-q) /(1-q)}>0 .
$$

Set $y(t)=\int_{\Omega} u \varphi_{1} d x$. Then, from (4.4) and (4.5), we have

$$
\frac{d y(t)}{d t} \geq M y^{p}(t)
$$

Integrating this inequality, we obtain

$$
y(t) \geq\left[\frac{1}{y_{0}^{1-p}-(p-1) M t}\right]^{1 /(p-1)}, \quad y_{0}=\int_{\Omega} u_{0} \varphi_{1} d x .
$$

Let $T_{M}^{0}=y_{0}^{1-p} /((p-1) M)$. It follows from (4.6) that there exists a maximal time $T_{m} \leq T_{M}^{0}$ such that

$$
\lim _{t \rightarrow T_{m}} y(t)=+\infty
$$

Consequently, we obtain

$$
\lim _{t \rightarrow T_{m}} \max _{x \in \Omega} u(x, t)=+\infty .
$$

This completes the proof of Theorem 4.3(i).

(ii) It is evident that Problem (4.1) has no positive global solution for $u_{0}(x)$ large enough. Hence, we have only to prove that Problem (4.1) has a global solution under the condition that $\lambda_{1}(\Omega) \geq C_{0}(n)$ and $u_{0}(x)$ is small enough. To this end, we denote by $u(x, t)$ a local solution of Problem (4.1). Since $\lambda_{1}(\Omega) \geq C_{0}(n)$, we know from Theorem 2.1(ii) that the problem

$$
\begin{cases}-\Delta v=v^{p}+v^{q}, & x \in \Omega, \\ v>0, & x \in \Omega, \\ v=0, & x \in \partial \Omega\end{cases}
$$

has at least one solution $v(x)$. It is obvious that $v(x)$ is a super-solution of Problem (4.1), provided that $u_{0}(x) \leq v(x)$. By Theorem 4.2, we have

$$
0 \leq u(x, t) \leq v(x) \text { for all }(x, t) \in \Omega \times(0,+\infty) .
$$

Consequently, $u(x, t)$ exists globally. This completes the proof of Theorem 4.3(ii). 
5. Cauchy Problem for Parabolic Equation. In this section, we will consider the Cauchy problem

$$
\begin{cases}\frac{\partial u}{\partial t}-\Delta u=u^{p}+u^{q}, & (x, t) \in \boldsymbol{R}^{n} \times(0, T), \\ u(x, 0)=u_{0}(x) \geq 0, & x \in \boldsymbol{R}^{n} .\end{cases}
$$

Here we only suppose $p, q>0$.

It is well-known that the following Cauchy problem

$$
\begin{cases}\frac{\partial u}{\partial t}-\Delta u=u^{p}, & (x, t) \in \boldsymbol{R}^{n} \times(0, T), \\ u(x, 0)=u_{0}(x) \geq 0, & x \in \boldsymbol{R}^{n}\end{cases}
$$

has been extensively studied since 1966, from the viewpoint of critical exponents of Fujita's type. Most of results on Problem (5.2) are collected in two excellent survey papers [10] and [11]. Roughly speaking, there are two critical exponents $p_{c_{1}}=1$ and $p_{c_{2}}=1+2 / n$ such that the following holds.

THEOREM 5.1. (i) If $0<p \leq p_{c_{1}}$, then any solution of Problem (5.2) exists globally. time.

(ii) If $p_{c_{1}}<p \leq p_{c_{2}}$, then any nontrivial solution of Problem (5.2) blows up in finite

(iii) If $p>p_{c_{2}}$, then Problem (5.2) has a global solution for small initial data, whereas has no nontrivial global solution for large initial data.

Theorem 5.1(i) is proved by Escobedo and Herrero [12]; (ii) and (iii) except for $p=p_{c_{2}}$ were proved by Fujita [13]; the result with respect to $p=p_{c_{2}}$ was obtained by several authors in [14-17].

Based on Theorem 5.1, we introduce the following definition.

DEFINITION 5.2. The exponent $p$ is said to belong to the global existence case if $0<p \leq p_{c_{1}}$ or $p>p_{c_{2}}$, and to belong to the blow up case if $p_{c_{1}}<p \leq p_{c_{2}}$.

Now, we turn to Problem (5.1). To our knowledge, there is no result for Problem (5.1) about the exponent of Fujita's type. Here, we try to give such a result. To this end, we denote

$$
\alpha=\min \{p, q\}, \quad \beta=\max \{p, q\} .
$$

Using the above notation, we now prove

THEOREM 5.3. (i) If $1<\alpha \leq 1+2 / n$, then any nontrivial solution of Problem (5.1) blows up in finite time.

(ii) If $\alpha>1+2 / n$, then Problem (5.1) has a global solution for small initial data and has no global solution for large initial data.

PRoOF. (i) If $1<\alpha \leq 1+2 / n$, then either $1<p \leq 1+2 / n$, or $1<q \leq 1+2 / n$. It follows from the comparison principle and Theorem 5.1(ii) that any nontrivial solution of Problem (5.1) blows up in finite time. 
(ii) If $\alpha>1+2 / n$, Problem (5.1) has no global solution for large initial data by the comparison principle together with Theorem 5.1(iii). In order to prove the global existence, we look for a global super-solution of the form

$$
U(x, t)=A(1+t)^{-\gamma} e^{-|x|^{2} /\{4(1+t)\}},
$$

where $A$ and $\gamma$ are positive numbers.

It is easy to check that $U(x, t)$ is a super-solution of Problem (5.1) only if

$$
\begin{aligned}
&-\gamma+\frac{n}{2} \geq A^{p-1}(1+t)^{1+\gamma-\gamma p} e^{-(p-1)|x|^{2} /\{4(1+t)\}} \\
&+A^{q-1}(1+t)^{1+\gamma-\gamma q} e^{-(q-1)|x|^{2} /\{4(1+t)\}} .
\end{aligned}
$$

Without loss of generality, we may suppose that

$$
q=\alpha=\min \{p, q\}>1 .
$$

If we choose $\gamma=1 /(q-1)$, then (5.3) becomes

$$
\begin{gathered}
-\frac{1}{q-1}+\frac{n}{2} \geq A^{p-1}(1+t)^{1+(1-p) /(q-1)} e^{-(p-1)|x|^{2} /\{4(1+t)\}} \\
+A^{q-1} e^{-(q-1)|x|^{2} /\{4(1+t)\}}
\end{gathered}
$$

Since $p \geq q>1,(5.4)$ holds only if

$$
-\frac{1}{q-1}+\frac{n}{2} \geq A^{q-1}\left(A^{p-q}+1\right) .
$$

By the assumption that $q=\alpha>1+2 / n$, we have

$$
-\frac{1}{q-1}+\frac{n}{2}>0
$$

This implies that (5.5) is in fact valid for $A$ small enough. Consequently, Problem (5.1) has a global solution for small initial data. The proof of Theorem 5.3 is completed.

A natural question which arises from Theorem 5.3 is: What would happen to $0<\alpha \leq 1$ ?

The case when $\alpha, \beta \in(0,1]$ is simple. In fact, by employing the same method as that used by Escobdo and Herrero in [12], one can easily prove

THEOREM 5.4. If $\alpha, \beta \in(0,1]$, then any solution of Problem (5.1) exists globally.

Theorems 5.3 and 5.4 seem to tell us that if both $p$ and $q$ belong to the global existence case, Problem (5.1) should have a global solution at least for small initial data. However, this is not always the case. For $\alpha \in(0,1]$ and $\beta>1+2 / n$, both $p$ and $q$ belong to the global existence case. Accordingly, it is reasonable to conjecture that Problem (5.1) should have a global solution at least for small initial data due to Theorems 5.3 and 5.4. However, this is not true. Indeed, we have the following blowing-up result.

THEOREM 5.5. If $\alpha \in(0,1]$ and $\beta>1$, then any nontrivial solution of Problem (5.1) blows up in finite time. 
Proof. If $0<\alpha<1$, then the nonlinearity $u^{p}+u^{q}$ is singular at 0 in the sense that it is not Lipschitz continuous. Hence the local existence result of Problem (5.1) is also to be proved. Accordingly, we proceed the proof in two steps.

Step 1. For any $0 \leq u_{0}(x) \in L^{\infty}\left(\boldsymbol{R}^{n}\right) \cap H^{1}\left(\boldsymbol{R}^{n}\right)$, Problem (5.1) has a positive solution defined on a maximal time interval $\left[0, T_{m}\right]$. Moreover, $T_{m}$ satisfies

$$
T_{m} \geq \frac{1}{M^{p}+M^{q}}, \quad M=\left\|u_{0}\right\|_{L^{\infty}\left(R^{n}\right)}+1 .
$$

Let $B_{r}(0)=\left\{x \in \boldsymbol{R}^{n}|| x \mid<r\right\}$ be an open ball, $\chi_{B_{r}}$ be the characteristic function of $B_{r}(0)$, i.e.,

$$
\chi_{B_{r}}= \begin{cases}1, & x \in B_{r}(0), \\ 0, & x \notin B_{r}(0) .\end{cases}
$$

We consider the initial-boundary value problem

$$
\begin{cases}\frac{\partial u_{r}}{\partial t}-\Delta u_{r}=u_{r}^{p}+u_{r}^{q}, & (x, t) \in B_{r}(0) \times(0, T), \\ u_{r}(x, t)=0, & (x, t) \in \partial B_{r}(0) \times(0, T), \\ u_{r}(x, 0)=u_{0}(x) \chi_{B_{r}}, & x \in B_{r}(0) .\end{cases}
$$

It follows from Theorem 4.1 together with Theorem 4.2 that Problem (5.6) has a unique positive solution $u_{r}(x, t)$ defined on a maximal interval $\left[0, T_{m}^{r}\right)$. In addition, the following properties hold:

(a) $u_{r}(x, t) \in L^{\infty}\left(B_{r}(0) \times(0, T)\right)$ for all $T<T_{m}^{r}$.

(b) $T_{m}^{r} \geq T_{0}=1 /\left(M^{p}+M^{q}\right)$ for all $r>0$.

(c) $u_{r}(x, t) \geq u_{s}(x, t)$ for any $r \geq s$, and $(x, t) \in B_{S}(0) \times\left(0, T_{0}\right)$.

(d) $0<u_{r}(x, t) \leq M$ for all $(x, t) \in B_{r}(0) \times\left[0, T_{0}\right]$.

By the properties (c), (d) and the regularity theory of parabolic equations, we conclude that, up to a subsequence, $u_{r}(x, t)$ converges uniformly to a function $u(x, t)$ on any bounded domain $\Omega \subset \boldsymbol{R}^{n}$ as $r \rightarrow+\infty$. In addition, $u(x, t)$ satisfies

$$
\begin{cases}\frac{\partial u}{\partial t}-\Delta u=u^{p}+u^{q}, & (x, t) \in \boldsymbol{R}^{n} \times\left(0, T_{0}\right), \\ u(x, t)>0, & (x, t) \in \boldsymbol{R}^{n} \times\left(0, T_{0}\right), \\ u(x, 0)=u_{0}(x), & x \in \boldsymbol{R}^{n} .\end{cases}
$$

Hence $u(x, t)$ is a local solution of Problem (5.1). The conclusion stated in Step 1 is proved.

Step 2. If $\alpha \in(0,1]$ and $\beta>1$, then any nontrivial solution of Problem (5.1) blows up in finite time.

Choose $\Omega_{0} \subset \boldsymbol{R}^{n}$ to be a bounded domain satisfying

$\left(H_{1}\right) \quad \lambda_{1}\left(\Omega_{0}\right)<((1-q) /(p-1))^{(p-1) /(p-q)}+((1-q) /(p-1))^{(q-1) /(p-q)}$, and

$\left(H_{2}\right) \quad$ there exists at least one point $x_{0} \in \Omega_{0}$ such that $u_{0}(x) \neq 0$.

Let $\chi \Omega_{0}$ be the characteristic function of $\Omega_{0}$. We prove the conclusion of Step 2 by contradiction. To this end, assume that Problem (5.1) has a global solution $u(x, t)$. It is 
obvious that $u(x, t)$ is a super-solution of the initial-boundary value problem

$$
\begin{cases}\frac{\partial v}{\partial t}-\Delta v=v^{p}+v^{q}, & (x, t) \in \Omega_{0} \times(0, T), \\ v(x, t)=0, & (x, t) \in \partial \Omega_{0} \times(0, T), \\ v(x, 0)=u_{0}(x) \chi \Omega_{0} & x \in \Omega_{0} .\end{cases}
$$

It follows from Theorems 4.1 and 4.2 that Problem (5.7) has a global solution. On the other hand, it follows from $\left(H_{1}\right)$ and Theorem 4.3(i) that Problem (5.7) has no global solution, which is a contradiction.

6. Parabolic Equation on Unbounded Domains. Let $\Omega \subset \boldsymbol{R}^{n}$ be a smooth unbounded domain in the sense of Definition 3.1. We consider the initial-boundary value problem

$$
\begin{cases}\frac{\partial u}{\partial t}-\Delta u=u^{p}+u^{q}, & (x, t) \in \Omega \times(0, T) \\ u(x, t)=0, & (x, t) \in \partial \Omega \times(0, T) \\ u(x, 0)=u_{0}(x) \geq 0, & x \in \Omega\end{cases}
$$

where $0 \leq q<1<p$.

Using the notation introduced in Section 3, the main result of this section can be stated as

THEOREM 6.1. (i) If $\rho(\Omega)=+\infty$, or $\rho(\Omega)<+\infty$ and $\lambda_{1}(\Omega)<c^{p-1}+c^{q-1}$, then any solution of Problem (6.1) blows up in finite time, and here $c=((1-q) /(p-$ 1) $)^{1 /(p-q)}$.

(ii) If $\rho(\Omega)<+\infty$ and $\lambda_{1}(\Omega) \geq C_{0}(n)$, then Problem (6.1) has a global solution for small initial data $u_{0}(x)$ and has no global solution for large initial data $u_{0}(x)$.

Since the proof of Theorem 6.1(i) is similar to that of Theorem 5.4, and the proof of Theorem 6.1(ii) is similar to that of Theorem 3.1(i), we omit them.

\section{REFERENCES}

[1] A. Ambrosetti, H. Brezis and G. Cerami, Combined effects of concave and convex nonlinearities in some elliptic problems, J. Funct. Anal. 122 (1994), 519-543.

[2] L. Boccardo, M. Escobedo and I. Peral, A Dirichlet problem involving critical exponent, Nonlinear Anal. 24 (1995), 1639-1648.

[ 3 ] Z. R. JIN, Solvability of Dirichlet problems for semi-linear elliptic equations on certain domains, Pacific J. Math. 176 (1996), 117-129.

[ 4 ] T. BATSCh AND M. Willem, On an elliptic equation with concave and convex nonlinearities, Proc. Amer. Math. Soc. 123 (1995), 3555-3561

[ 5 ] X. CABRe And P. Majer, Truncation of nonlinearities in some supercritical elliptic problems, C. R. Acad. Sci. Paris Sér. I Math. 322 (1996), 1157-1162.

[ 6 ] T. Cazenave, F. Dickstein And M. Escobedo, A semi-linear heat equation with concave-convex nonlinearity, Rend. Mat. Appl.(7) 19(1999), 211-242.

[ 7 ] P. Souplet, Decay of heat semigroup in $L^{\infty}$ and applications to nonlinear parabolic problems in unbounded domains, J. Funct. Anal. 173 (2000), 343-360. 
[ 8 ] M. J. Esteban And P. L. LiOnS, Existence and nonexistence results for semilinear elliptic problems in unbounded domains, Proc. Roy. Soc. Edinburgh Sect. A 93 (1982), 1-14.

[9] P. Souplet, Geometry of unbounded domains, Poincare inequalities, and stability in semilinear parabolic equations, Comm. Partial Differential Equations 24 (1999), 951-973.

[10] H. A. LEVINE, The role of critical exponents in blow up theorems, SIAM Rev. 32 (1990), 262-288.

[11] K. Deng And H. A. Levine, The role of critical exponents in blow up theorems, The sequel, J. Math. Anal. Appl. 243 (2000), 85-126.

[12] M. Escobedo AND M. A. Herrero, Boundedness and blow up for a semilinear reaction-diffusion system, J. Differential Equations 89 (1991), 176-202.

[13] H. FujitA, On the blowing up of solution of Cauchy problem for $u_{t}=\Delta u+u^{1+\alpha}$, J. Fac. Sci. Univ. Tokyo Sect. IA Math. 16 (1966), 105-113.

[14] D. J. Aronson and H. F. Weinberger, Multidimensional nonlinear diffusion arising in population genetics, Adv. Math. 30(1978), 33-76.

[15] K. HayakaWA, On nonexistence of global solutions of some semilinear parabolic equations, Proc. Japan Acad. 49 (1973), 503-525.

[16] K. Kobayashi, T. Siaro and H. TAnaka, On the blowing up problem for semilinear heat equations, J. Math. Soc. Japan 29 (1997), 407-424.

[17] F. B. WESSLER, Existence and nonexistence of global solutions for a semilinear heat equation, Israel J. Math. 38 (1981), 29-40.

[18] H. Brezis and L. Nirenberg, Positive solutions of nonlinear elliptic equations involving critical Sobolev exponents, Comm. Pure Appl. Math. 36 (1983), 437-477.

[19] A. Ambrosetti And P. H. Rabinowitz, Dual variational methods in critial point theory and applications, J. Funct. Anal. 14 (1973), 349-381.

[20] M. Willem, Minmax Theorems, Progr. Nonlinear Differential Equations Appl., 24, Birkhauser, Boston Inc., Boston, Mass., 1996.

[21] QIUYi DAI AND YongGeng GU, A short note on quenching phenomena for semilinear parabolic equations, J. Differential Equations 137 (1997), 240-250.

[22] QIUYi DAI AND XIANZHONG ZENG, The quenching phenomena for the Cauchy problem of semilinear parabolic equations, J. Differential Equations 175 (2001), 163-174.

[23] Qiuyi Dai And Yonggeng Gu, Some results on quenching problem and its applications, Acta Math. Sinica 46 (2003), 985-992.

[24] J. L. KAZDAN AND R. J. KRAMER, Invariant criteria for existence of solutions to second order quasilinear elliptic equations, Comm. Pure Appl. Math. 31 (1978), 619-645.

[25] H. A. Levine, Advances in quenching, Nonlinear diffusion equations and their equilibrium states, 3 (Gregynog, 1989), 319-346, Progr. Nonlinear Differential Equations Appl. 7, Bikhauser Boston, Boston, Mass., 1992.

DEPARTMENT OF MATHEMATICS

HUNAN NORMAL UNIVERSITY

Changsha Hunan 410081

P. R. CHINA 